01

\title{
Феномен статистической неустойчивости систем третьего типа-complexity
}

\author{
() В.В. Еськов, Т.В. Гавриленко, В.М. Еськов, Ю.В. Вохмина
}

Сургутский государственный университет, 626415 Сургут, Россия

e-mail: valery.eskov@gmail.com

(Поступило в Редакцию 29 декабря 2016 г.)

\begin{abstract}
Проблема реальности и особых свойств систем третьего типа представлена в рамках новой теории хаоса-самоорганизации. Фактически ставится глобальная проблема о возможности существования стационарных режимов у гомеостатических систем. К этим системам относятся не только медицинские и биологические системы, но и динамика метеопараметров, параметров окружающей среды, в которой находится человек. В рамках нового подхода дается новое определение стационарных режимов гомеостатических систем-complexity.
\end{abstract}

DOI: 10.21883/JTF.2017.11.45117.2158

\section{Введение}

До настоящего времени считалось, что регуляторные динамические системы в биологии и медицине, а также метеопараметры, климат можно описывать в рамках стохастики или хотя бы в рамках моделей динамического хаоса. Имеются также многочисленные попытки описывать такие особые системы третьего типа (СTT) в рамках фрактальной размерности, мультифракталами и т.д. В последнем были убеждены два нобелевских лауреата (I.R. Prigogine [1] и M. Gell-Mann [2]) и J.A. Wheeler [3], что представлено в целом ряде их публикаций. Однако действительность оказалась иной любые биомеханические и многие параметры других регуляторных систем человека при организации движения, работы сердца, биоэлектрической активности мозга человека и т.д. не могут генерировать инвариантность мер или стремление автокорреляционных функций $A(t)$ к нулю с ростом времени $t$. Оказалось, что СТТ-complexity имеют особый хаос в виде непрерывно изменяющихся функций распределения $f(x)$ [4-8]. Поскольку для СТТ нет выполнения $A(t) \rightarrow 0$, константы Ляпунова меняют знак непрерывно, нет инвариантности мер (нет свойства перемешивания), хаос СТТ не описывается аттрактором Лоренца. Динамический хаос Лоренца-Арнольда не моделирует СТТ [8-12].

Стохастические (и хаотические) подходы сейчас пытаются применять и в описании параметров климата (долгосрочный прогноз) и метеопараметров окружающей среды (в физике атмосферы, например). Как отмечается в ряде дискуссионных работ $[13,14]$ о статистической устойчивости для разнообразных физических процессов, эта гипотеза об устойчивости не подтверждается именно при изучении динамики ряда физических систем [13] и биосистем [4-8,10,15-18]. В таких эффектах неустойчивости наблюдается нарушение устойчивости выборочного среднего, моды или выборочного среднеквадратического отклонения (СКО) или выборочных моментов более высокого порядка. Тогда возникает другая проблема неустойчивости - это неустойчивость характеристик СТТ в виде параметров вектора состояния таких complexity $x=x(t)=\left(x_{1}, x_{2}, \ldots, x_{m}\right)^{T}$ по всем координатам $x_{i}$. Для СТТ имеются все-таки некоторые стохастические закономерности, но особый хаос СТТ (эмерджентных систем по J.A. Wheeler [3]) не является объектом современной науки (именно это высказал I.R. Prigogine в своей известной работе [1]). Именно это пытался представить в ряде своих публикаций И.И. Горбань [13], но проблема выходит за рамки неустойчивости при больших интервалах наблюдения [13]. Сейчас речь идет о глобальной неустойчивости гомеостатических систем, к которым относятся не только биосистемы, но и климат, метеопараметры и экопараметры среды обитания человека [4-9,11,12,20].

Одновременно ряд авторов подчеркивает связь между статистической устойчивостью (у нас, наоборот, неустойчивостью) процесса и его спектром [1,4]. При этом особым образом подчеркивается связь корреляционных функций (КФ) и спектральной плотностью сигнала (СПС). Например, подчеркивается связь КФ и СПС $S_{x}(t)$ для стационарного процесса $x(t)$ интегральным преобразованием Винера-Хинчина [13].

В нашем исследовании в области биомеханики и изучения метеопараметров среды для СТТ мы показываем, что характер этих неопределенностей различен, в биомеханике мы имеем особый тип хаоса - это хаос (непрерывное и непрогнозируемое изменение) любых статистических характеристик любых живых систем, которые мы обозначаем как гомеостатические системы (или СТТ), complexity [4-9,19-21].

\section{Количественная интерпретация эффекта статистической неустойчивости в биомеханике}

Общеизвестно, что понятие статистической устойчивости процессов сводится к стабилизации измеряемых 
характеристик процессов (выборочного среднего, СКО, статистической дисперсии - СД, выборочных моментов более высокого порядка) при увеличении интервала усреднения усредненных величин этих процессов. Традиционно в математической статистике вводится критерий статистической устойчивости по отношению к выборочному среднему, для случайного процесса $x(t)$ с выборочным средним $Y_{N}$. Процесс считается устойчивым, если при устремлении объема выборки $N$ к бесконечности параметр статистической неустойчивости $\gamma_{N}=M\left[\bar{D}_{Y_{N}}\right] / \bar{D}_{x_{N}}$ стремится к нулю.

Однако все процедуры расчета статистических величин и их оценок могут быть выполнены, если имеется выполнение двух принципиальных условий.

Во-первых, процесс не должен участвовать в эволюции своих параметров, иначе все измеряемые величины будут изменяться в рамках каких-либо законов или даже хаотически (но без флуктуаций вокруг некоторых средних величин). В этом случае выборочное среднее, СКО и выборочные моменты более высокого порядка будут закономерно и целенаправленно изменяться (дрейфовать), например, в сторону их увеличения или уменьшения. Тогда все вышеприведенные расчеты теряют смысл, и, возможно, этим объясняется неустойчивость $x_{i}$ на больших интервалах наблюдений у И.И. Горбаня [13]. Отметим, что базовыми свойствами любой живой системы является именно эволюция любых параметров, например компонент $x_{i}$ всего вектора состояния системы $x=x(t)=\left(x_{1}, x_{2}, \ldots, x_{m}\right)^{T}$ в $m$-мерном фазовом пространстве состояний (ФПС). По этой причине мы не можем использовать всю идеологию статистики и методы оценки статистической устойчивости процессов для живых систем, в частности, для биомеханических систем.

Во-вторых, мы должны подтвердить возможность параметрических распределений, для которых вообще возможен расчет выборочного среднего, $\gamma_{N}$, выборочного СКО и др. статистических параметров функций распределения $f(x)$. Нами установлено, что для любого биомеханического движения при попытках повторения измерений получаются выборки $x_{1}, x_{2}, \ldots$, которые только в $1-2 \%$ случаев можно описывать параметрическими законами распределения. В 99\% случаев мы имеем непараметрические распределения. Одновременно с учетом возможности эволюции СТТ задача в целом усложняется многократно, так как сами $f(x)$ и др. характеристики СТТ могут хаотически изменяться. Именно это является базовым критерием гомеостатических CTT-complexity, и это сразу выводит complexity за пределы традиционной статистики. Однако отказываться от статистики в изучении биосистем все-таки еще рано и нецелесообразно [4-9,11-20].

Очевидно, что в анализе особенностей динамики поведения сигнала $x(t)$ в биомеханике большую роль играет не только специфика СКО, дисперсий и выборочных моментов более высокого порядка, но и СПС, корреляционный (автокорреляционный) анализ. Именно
Таблица 1. Матрица парного сравнения выборок треморограмм испытуемого ГДВ (число повторов $N=15$ ), использовался критерий Вилкоксона (уровень значимости $p<0.05$, число совпадений $k_{1}=4$ )

\begin{tabular}{r|c|c|c|c|c|c|c|c|c|c|c|c|c|c|c}
\hline & 1 & 2 & 3 & 4 & 5 & 6 & 7 & 8 & 9 & 10 & 11 & 12 & 13 & 14 & 15 \\
\hline 1 & & .00 & .00 & .00 & .00 & .00 & .00 & .00 & .00 & .00 & .00 & .00 & .00 & .00 & .00 \\
2 & .00 & & .00 & .00 & .00 & .00 & .00 & .00 & .00 & .00 & .07 & .00 & .00 & .00 & .00 \\
3 & .00 & .00 & & .00 & .00 & .00 & .00 & .00 & .00 & .00 & .00 & .00 & .00 & .00 & .00 \\
4 & .00 & .00 & .00 & & .00 & .00 & .00 & .00 & .00 & .82 & .00 & .00 & .00 & .00 & .00 \\
5 & .00 & .00 & .00 & .00 & & .00 & .00 & .00 & .00 & .00 & .00 & .00 & .00 & .00 & .00 \\
6 & .00 & .00 & .00 & .00 & .00 & & .00 & .00 & .00 & .00 & .00 & .00 & .00 & .00 & .57 \\
7 & .00 & .00 & .00 & .00 & .00 & .00 & & .00 & .00 & .00 & .00 & .00 & .95 & .00 & .00 \\
8 & .00 & .00 & .00 & .00 & .00 & .00 & .00 & & .00 & .00 & .00 & .00 & .00 & .00 & .00 \\
9 & .00 & .00 & .00 & .00 & .00 & .00 & .00 & .00 & & .00 & .00 & .00 & .00 & .00 & .00 \\
10 & .00 & .00 & .00 & .82 & .00 & .00 & .00 & .00 & .00 & & .00 & .00 & .00 & .00 & .00 \\
11 & .00 & .07 & .00 & .00 & .00 & .00 & .00 & .00 & .00 & .00 & & .00 & .00 & .00 & .00 \\
12 & .00 & .00 & .00 & .00 & .00 & .00 & .00 & .00 & .00 & .00 & .00 & & .00 & .00 & .00 \\
13 & .00 & .00 & .00 & .00 & .00 & .00 & .95 & .00 & .00 & .00 & .00 & .00 & & .00 & .00 \\
14 & .00 & .00 & .00 & .00 & .00 & .00 & .00 & .00 & .00 & .00 & .00 & .00 & .00 & & .00 \\
15 & .00 & .00 & .00 & .00 & .00 & .57 & .00 & .00 & .00 & .00 & .00 & .00 & .00 & .00 &
\end{tabular}

этой проблеме мы и посвящаем наши усилия в отношении различных характеристик систем живой природы (на примере биомеханических параметров) и неживой природы (метеопараметры). Сразу отметим, что мы сейчас в биомеханике говорим об анализе динамики поведения координаты $x_{1}(t)$ - положения части тела испытуемого. При треморе или теппинге это координата пальца с металлической пластиной по отношению к токовихревому датчику. За счет дискретизации измерения имеем указанную последовательность $x_{i}$, которая у нас для $x_{1}$ (перемещение конечности в пространстве) дает набор $x_{1 k}$, где $k-1,2, \ldots, n$ и $x_{2}=d x_{1} / d t-$ скорость изменения $x_{1}(t)$, а также в ряде случаев мы используем и $x_{3}=d x_{2} / d t$ - ускорение изменения $x_{1}(t)$ [4-9,18-22].

Все эти значения $x_{i}$ у нас были дискретные (обычно период квантования в измерениях был $\Delta t=10 \mathrm{~ms}$ ). Координаты такого трехмерного вектора состояния биомеханической системы $x=x(t)=\left(x_{1}, x_{2}, x_{3}\right)^{T}$ непрерывно изменяются в трехмерном фазовом пространстве состояний и образуют некоторые ограниченные последовательности (обычно эпоха наблюдения $T=5 \mathrm{~s}$ и тогда $n=500$ ), которые мы при повторных (подряд) регистрациях определяли как выборки. Для этих выборок (по $n$ точек в каждой) мы рассчитывали статистические характеристики у всех наборов $x_{1}(t)$ при их парном сравнении. При этом строились матрицы парных сравнений, которые при 15 подряд получаемых выборках $x_{i}(t)$ имели всего 105 независимых пар сравнения, и такую закономерность можно получить только в рамках компартментно-кластерного моделирования [19,22-26].

Для всех биомеханических систем (а это более 1000 выборок треморограмм - ТМГ и теппинграмм ТПГ) наблюдается устойчивая закономерность: получаемые выборки могут удовлетворять закону нормального 
распределения не более чем в $1 \%$ случаев (все остальные - непараметрические распределения) [4-9,14]. Сейчас мы используем матрицы парного сравнения выборок, получаемых от одного и того же испытуемого (подряд), который якобы находится в одинаковом физиологическом состоянии. В биологии это обозначают гомеостазом [2-9,19-25]. В табл. 1 мы представляем типичный пример такой матрицы (при $n=500$ точек и числе повторов измерений выборок $N=15$ ). Очевидно, что число $k$ пар совпадений выборок для одного испытуемого в табл. 1 невелико, $k=4$. Из тысячи таких повторов для тремора мы получили в среднем $\langle k\rangle=5 \%$. Повторение без повторений Н.А. Бернштейна [26] для тремора закономерно: только $k \approx 5 \%$ (число пар треморограмм) можно отнести к одной генеральной совокупности, остальные выборки $x_{1}(t)$ разные.

Такая закономерность касается не только треморограмм и теппинграмм (биомеханических параметров испытуемых), но и всех других параметров, которые широко сейчас используются в физиологии, биологии, медицине, психологии, в глобальном изучении гомеостаза организма человека: выборок кардиоинтервалов, электромиограмм, электроэнцефалограмм, электронейрограмм, биохимических параметров крови и многих других параметров организма человека, которые очень широко и повсеместно используются в биологии, медицине, психологии и различных науках о живом. Очевидно, если $k<30 \%$, то мы имеем уникальные процессы, и любая выборка получается в биомедицине „без повторений“. Тогда возникает закономерный вопрос: о какой статистике может сейчас идти речь в биологии и медицине (если любую выборку произвольно мы не можем повторить!)? С чем работает современная медицина? Можно ли к ней применять критерии, которые широко используются в технике, физике и химии?

Поскольку на возможность такого исхода при анализе измерений параметра организма человека впервые высказал Н.А. Бернштейн (его гипотеза о „повторении без повторения“ [26]), его детальное исследование в психофизиологии и в биомеханике при изучении механизмов организации движений привело нас к открытию эффекта Еськова-Зинченко (в психофизиологии). В более широком смысле эффект Еськова-Зинченко включает общее свойство любых гомеостатических систем, которые демонстрируют статистическую неустойчивость в аспекте непрерывного (хаотического) изменения $f(x), A(t)$, СПС и различных других характеристик выборок любых компонент $x_{i}$ всего вектора $x(t)$ [2-9,12-20,27-31].

Как оказалось, неустойчивость СТТ - это более широкое понятие, и оно охватывает все гомеостатические системы в психологии, экологии, биологии и медицине (включая и параметры гомеостаза всей биосферы Земли). Подчеркнем, что сейчас для нас очевидно: для естествознания необходимо введение нового понятия в виде гомеостатического регулирования и гомеостатических систем в целом. Об этом пытался сказать I.R. Prigogine [1], говоря о живых системах как об
Таблица 2. Матрица парного сравнения выборок теппинграмм испытуемого ГДВ (число повторов $N=15$ ), использовался критерий Вилкоксона (уровень значимости $p<0.05$, число совпадений $k_{2}=17$ )

\begin{tabular}{c|c|c|c|c|c|c|c|c|c|c|c|c|c|c|c}
\hline & 1 & 2 & 3 & 4 & 5 & 6 & 7 & 8 & 9 & 10 & 11 & 12 & 13 & 14 & 15 \\
\hline 1 & & .56 & .00 & .00 & .00 & .00 & .00 & .00 & .00 & .00 & .00 & .00 & .00 & .00 & .00 \\
2 & .56 & & .00 & .00 & .00 & .00 & .00 & .00 & .00 & .00 & .00 & .00 & .00 & .00 & .00 \\
3 & .00 & .00 & & .00 & .01 & .04 & .00 & .00 & .00 & .00 & .00 & .00 & .00 & .00 & .00 \\
4 & .00 & .00 & .00 & & .73 & .00 & .02 & .00 & .00 & .00 & .00 & .00 & .00 & .00 & .00 \\
5 & .00 & .00 & .01 & .73 & & .00 & .05 & .00 & .00 & .00 & .00 & .00 & .00 & .00 & .00 \\
6 & .00 & .00 & .04 & .00 & .00 & & .00 & .00 & .00 & .00 & .00 & .00 & .00 & .00 & .00 \\
7 & .00 & .00 & .00 & .02 & .05 & .00 & & .00 & .00 & .02 & .00 & .00 & .00 &. $\mathbf{1 2}$ & .00 \\
8 & .00 & .00 & .00 & .00 & .00 & .00 & .00 & & .44 & .01 & .72 & .00 &. $\mathbf{6 2}$ & $\mathbf{. 1 1}$ &. $\mathbf{7 0}$ \\
9 & .00 & .00 & .00 & .00 & .00 & .00 & .00 & .44 & & .00 & .96 & .00 & .03 & .00 &. $\mathbf{4 8}$ \\
10 & .00 & .00 & .00 & .00 & .00 & .00 & .02 & .01 & .00 & & .01 & .00 &. $\mathbf{1 1}$ &. $\mathbf{3 3}$ & .00 \\
11 & .00 & .00 & .00 & .00 & .00 & .00 & .00 & .72 & .96 & .01 & & .00 &. $\mathbf{2 8}$ & .03 &. $\mathbf{3 6}$ \\
12 & .00 & .00 & .00 & .00 & .00 & .00 & .00 & .00 & .00 & .00 & .00 & & .00 & .00 & .00 \\
13 & .00 & .00 & .00 & .00 & .00 & .00 & .00 & .62 & .03 & .11 & .28 & .00 & & $\mathbf{. 4 8}$ &. $\mathbf{1 6}$ \\
14 & .00 & .00 & .00 & .00 & .00 & .00 & .12 & .11 & .00 & .33 & .03 & .00 & .48 & & $\mathbf{. 0 9}$ \\
15 & .00 & .00 & .00 & .00 & .00 & .00 & .00 & .70 & .48 & .00 & .36 & .00 & .16 & .09 &
\end{tabular}

Таблица 3. Матрица парного сравнения 15 выборок коэффициентов автокорреляций $A(t)$ треморограмм одного испытуемого ГДВ при повторных экспериментах $\left(k_{3}=27\right)$, по критерию Вилкоксона (для непараметрического распределения)

\begin{tabular}{c|c|c|c|c|c|c|c|c|c|c|c|c|c|c|c}
\hline & 1 & 2 & 3 & 4 & 5 & 6 & 7 & 8 & 9 & 10 & 11 & 12 & 13 & 14 & 15 \\
\hline 1 & & .01 & .00 &. $\mathbf{4 3}$ & .00 &. $\mathbf{1 0}$ & .00 & .00 & .00 & .00 & .00 & .00 &. $\mathbf{2 3}$ & .04 & .00 \\
2 & .01 & & .00 &. $\mathbf{3 8}$ & .00 &. $\mathbf{4 3}$ & $\mathbf{. 0 5}$ &. $\mathbf{0 7}$ & .00 &. $\mathbf{7 7}$ & .00 & .00 &. $\mathbf{0 9}$ & .00 & .00 \\
3 & .00 & .00 & & .00 & .00 & .00 & .01 & .01 & .00 & .00 & .00 & .00 & .00 & .00 & .00 \\
4 & .43 & .38 & .00 & & .00 &. $\mathbf{1 3}$ & .00 & .02 & .00 &. $\mathbf{1 1}$ & .00 & .00 &. $\mathbf{4 1}$ & .00 & .00 \\
5 & .00 & .00 & .00 & .00 & & .00 & .00 & .00 & .00 & .00 & .00 &. $\mathbf{0 7}$ & .00 & .00 & .00 \\
6 & .10 & .43 & .00 & .13 & .00 & & $\mathbf{. 1 0}$ &. $\mathbf{1 0}$ & .00 &. $\mathbf{6 6}$ & .00 & .00 &. $\mathbf{1 0}$ & .00 & .00 \\
7 & .00 & .05 & .01 & .00 & .00 & .10 & &. $\mathbf{4 7}$ & .00 &. $\mathbf{1 3}$ & .00 & .00 & .00 & .00 & .00 \\
8 & .00 & .07 & .01 & .02 & .00 & .10 & .47 & & .00 &. $\mathbf{6 3}$ & .00 & .00 & .00 & .00 & .00 \\
9 & .00 & .00 & .00 & .00 & .00 & .00 & .00 & .00 & & .00 & $\mathbf{. 8 3}$ & $\mathbf{. 0 8}$ & .00 & .05 &. $\mathbf{4 7}$ \\
10 & .00 & .77 & .00 & .11 & .00 & .66 & .13 & .63 & .00 & & .00 & .00 & .02 & .00 & .00 \\
11 & .00 & .00 & .00 & .00 & .00 & .00 & .00 & .00 & .83 & .00 & & $\mathbf{. 1 3}$ & .00 & $\mathbf{. 3 1}$ &. $\mathbf{4 5}$ \\
12 & .00 & .00 & .00 & .00 & .07 & .00 & .00 & .00 & .08 & .00 & .13 & & .00 & .00 & .03 \\
13 & .23 & .09 & .00 & .41 & .00 & .10 & .00 & .00 & .00 & .02 & .00 & .00 & & .00 & .00 \\
14 & .04 & .00 & .00 & .00 & .00 & .00 & .00 & .00 & .05 & .00 & .31 & .00 & .00 & &. $\mathbf{2 6}$ \\
15 & .00 & .00 & .00 & .00 & .00 & .00 & .00 & .00 & .47 & .00 & .45 & .03 & .00 & .26 &
\end{tabular}

уникальных системах (не объектах современной науки). Однако нобелевский лауреат с двумя другими своими коллегами (J.A. Wheeler и M. Gell-Mann) оставались в плену убеждения, что СТТ (биосистемы - complexity) являются объектом динамического хаоса. Это убеждение оказалось ошибочным, так как хаос биосистем в гомеостазе не демонстрирует аттракторы Лоренца [2-9,14-20]. Одновременно мы переходим и к другому фундаментальному вопросу естествознания: существует ли вообще возможность описания CTT-complexity (гомеостатических систем) в рамках физико-математического подхода в целом и где границы применимости современной ма- 
Таблица 4. Матрица парного сравнения выборок температуры $T$ за месяц апрель 1991-2005 гг., использовался критерий Вилкоксона (уровень значимости $p<0.05$, число совпадений $k=19$ )

\begin{tabular}{|c|c|c|c|c|c|c|c|c|c|c|c|c|c|c|c|}
\hline Годы & 1991 & 1992 & 1993 & 1994 & 1995 & 1996 & 1997 & 1998 & 1999 & 2000 & 2001 & 2002 & 2003 & 2004 & 2005 \\
\hline 1991 & & .00 & .01 & .00 & .00 & .00 & .00 & .00 & .00 & .00 & .00 & .00 & .00 & .00 & .00 \\
\hline 1991 & .00 & & .00 & .90 & .53 & .00 & .00 & .02 & .00 & .19 & .99 & .00 & .00 & .07 & .00 \\
\hline 1991 & .01 & .00 & & .00 & .00 & .00 & .00 & .00 & .00 & .00 & .00 & .00 & .00 & .00 & .00 \\
\hline 1991 & .00 & .90 & .00 & & .42 & .00 & .00 & .01 & .00 & .06 & .95 & .00 & .00 & .04 & .00 \\
\hline 1991 & .00 & .53 & .00 & .42 & & .00 & .00 & .00 & .00 & .02 & .23 & .00 & .00 & .01 & .00 \\
\hline 1991 & .00 & .00 & .00 & .00 & .00 & & .01 & .00 & .00 & .00 & .00 & .00 & .68 & .00 & .02 \\
\hline 1991 & .00 & .00 & .00 & .00 & .00 & .01 & & .00 & .39 & .00 & .00 & .00 & .19 & .00 & .42 \\
\hline 1991 & .00 & .02 & .00 & .01 & .00 & .00 & .00 & & .00 & .20 & .05 & .00 & .00 & .95 & .00 \\
\hline 1991 & .00 & .00 & .00 & .00 & .00 & .00 & .39 & .00 & & .00 & .00 & .00 & .01 & .00 & .58 \\
\hline 1991 & .00 & .19 & .00 & .06 & .02 & .00 & .00 & .20 & .00 & & .10 & .00 & .00 & .75 & .00 \\
\hline 1991 & .00 & .99 & .00 & .95 & .23 & .00 & .00 & .05 & .00 & .10 & & .00 & .00 & .11 & .00 \\
\hline 1991 & .00 & .00 & .00 & .00 & .00 & .00 & .00 & .00 & .00 & .00 & .00 & & .00 & .00 & .00 \\
\hline 1991 & .00 & .00 & .00 & .00 & .00 & .68 & .19 & .00 & .01 & .00 & .00 & .00 & & .00 & .03 \\
\hline 1991 & .00 & .07 & .00 & .04 & .01 & .00 & .00 & .95 & .00 & .75 & .11 & .00 & .00 & & .00 \\
\hline 1991 & .00 & .00 & .00 & .00 & .00 & .02 & .42 & .00 & .58 & .00 & .00 & .00 & .03 & .00 & \\
\hline
\end{tabular}

тематики и физики в изучении гомеостатических систем (CTT-complexity) [2-9,11-20].

Ответы на эти вопросы легко получить, если подробно изучить эффект Еськова-Зинченко в биомеханике. Отметим, что Н.А. Бернштейн в 1947 г. только обратил внимание на возможность „без повторений“ в биомеханике, но количественную интерпретацию дали мы уже в XXI веке [4-12,14-22]. Фактически, речь идет о введении аналога принципа неопределенности Гейзенберга (который широко используется в квантовой механике) в биомеханику и во всю медико-биологическую науку [4-11,15,28-31]. Аналог принципа неопределенности Гейзенберга в биологии и медицине действительно позволяет нам оперировать с более устойчивыми характеристиками (объемами квазиаттракторов и координатами их центров в ФПС), чем традиционные стохастические характеристики [22-25,27,29].

Иллюстрация этому тезису очень проста, если мы будем статистически анализировать выборки, которые получаются у одного и того же человека при регистрации его биомеханических параметров: произвольных, не произвольных движений (в нашем сообщении) и всех других параметров гомеостаза. При получении подряд 15 выборок якобы произвольных движений теппинграмм - ТПГ (сравните с матрицей в табл. 1 для ТМГ, где $\langle k\rangle \approx 5 \%$ ) от одного человека (все его физиологические характеристики при этом якобы находятся в неизменном состоянии) мы можем построить матрицу парных сравнений 15 получаемых подряд (в одном эксперименте) выборок теппинграмм. Это представлено для примера в табл. 2, где демонстрируется критерий Вилкоксона $p$ при парном сравнении всех 15 выборок (общее число пар сравнений $x_{i}=105$ ).

Появляется полная неопределенность в получаемых выборках ТМГ и ТПГ, так как получить две одинаковые выборки, их одинаковые две статистические функции распределения $f(x)$ весьма затруднительно. Одновременно и любые СПС, и автокорреляции $A(t)$ невозможно произвольно повторить. Всегда СПС и $A(t)$ у треморограмм будут без повторений. Биосистемы не являются статистически устойчивыми системами, их статистические функции непрерывно и хаотически изменяются [27,29-31].

В табл. 3 мы демонстрируем динамику поведения значения автокорреляций $A(t)$ при подряд 15 регистрациях тремора (у одного и того же испытуемого в неизменном состоянии). Оказывается, что, как и для статистических функций распределения $f(x)$, невозможно получить подряд (произвольно) две одинаковые $A(t)$. В табл. 3 для испытуемого ГДА мы получаем некоторое число пар совпадений $A(t)$, но это число не превышает $30 \%$. Более того, могут совпадать последние функции $A(t)$ с начальными $A(t)$, что в динамическом хаосе не должно наблюдаться (автокорреляция должна стремиться к нулю). Аналогичные закономерности наблюдаются и для СПС.

\section{Новая интерпретация гомеостатических систем}

Для любых гомеостатических систем (complexity) мы получаем несовпадения для СПС, $f(x), A(t)$ сигнала $x_{1}(t)$. Все эти таблицы $(1,2,3)$ являются типичными (средними) примерами для всех испытуемых, их ТМГ и ТПГ при 15 повторах. Более того, мы делали повторы измерений по 15 сериям из 15 треморограмм в каждой серии (всего 225 выборок ТМГ и ТПГ у одного и того же испытуемого при неизменности его гомеостаза). Оказалось, что для тремора число совпадений пар выборок треморограмм $k \approx 5 \%$, а для СПС $k \approx 20 \%$. Иными словами, табл. 1-3 являются характерными примерами 
для треморограмм любого здорового человека, находящегося в спокойном состоянии, но изменение гомеостаза одновременно и закономерно вызывает изменение $k$.

Такое состояние мы сейчас называем гомеостазом. Гомеостатическими свойствами обладают не только параметры организма, но и метеопараметры, параметры климата. Такая динамика наблюдается в краткосрочном варианте для метеопараметров $x_{1}=P-$ атмосферное давление, $x_{2}=T$ - температура воздуха, $x_{3}=R-$ относительная влажность воздуха. Все эти три параметра в таком трехмерном фазовом пространстве совершают непрерывное и хаотическое движение в течение суток, месяцев, года. Для этих трех координат мы можем представить матрицы парного сравнения выборок, которые так же, как и у ТМГ и ТПГ, демонстрируют небольшое число $k$ совпадений $[8,16,19,20]$.

В качестве примера мы представляем характерную матрицу парного сравнения выборок температур $T$ для 10 одинаковых месяцев (апрель), взятых подряд для 15 лет сравнения в ХМАО-Югре (Север РФ). Очевидно, что число $k$ пар сравнений выборок для $T$ невелико. Иными словами, выборки температур ведут себя так, как ТМГ у всех испытуемых. Организация хаоса в регуляции движений подобна реуляции метеопараметров среды (аналогично и для $P, R$ ) и она показывает отсутствие динамического хаоса Лоренца [2-9,15-20].

В табл. 4 приведен пример матрицы парного сравнения температур для апреля в Югре (Россия). Как видно из этой таблицы, число пар $k=19$ при сравнении колебаний $T$ для 15 апрелей за 15 лет. Аналогичные результаты демонстрируют летний месяц июль и осенний месяц октябрь. В целом доля стохастики (в виде $k$ ) снижается весной и осенью и нарастает летом и зимой.

Таким образом, мы сейчас можем говорить о гомеостатичном регулировании не только функций организма человека, но и о гомеостазе метеопараметров. Во всех этих случаях мы имеем хаос статистических характеристик и отсутствие аттракторов Лоренца.

\section{Выводы}

1. Гомеостатические системы характеризуются статистической неустойчивостью, так как их выборки $x_{i}(t)$, их функции распределения $f(x)$, СПС, автокорреляции непрерывно и хаотически изменяются. Однако это не динамический хаос Лоренца.

2. Хаос гомеостатических систем имеет некоторые статистические закономерности, которые проявляются в матрицах парных сравнений выборок. Непроизвольный тремор имеет обычно $k \approx 5 \%$, а произвольный теппинг - около $17 \%$ совпадений выборок. Эти особые системы третьего типа ТT (complexity) имеют некоторую „статичность“ в виде неизменности числа пар совпадений выборок как ТМГ, ТПГ, так и метеопараметров среды $(P, T, R)$.

\section{Список литературы}

[1] Prigogine I. The End of Certainty: Time, Chaos, and the New Laws of Nature. Free Press. 1996. P. 64.

[2] Gell-Mann M. // Complexity. 1997. Vol. 3. N 1. P. 13-19.

[3] Wheeler J.A. Information, physics, quantum: the search for links. In Feyman and Computation: Exploring the Limits of Computers / Ed by A.J.G. Hey. Cambridge, MA: Perseus Books, 1999. P. 309.

[4] Betelin V.B., Eskov V.M., Galkin V.A., Gavrilenko T.V. // Doklady Mathematics. 2017. Vol. 472. N 6.

[5] Eskov V.M., Gavrilenko T.V., Vokhmina Y.V., Zimin M.I., Filatov M.A. // Measurement Techniques. 2014. Vol. 57. N 6. P. 720-724.

[6] Eskov V.M., Eskov V.V., Gavrilenko T.V., Zimin M.I. // Moscow University Physics Bulletin. 2014. Vol. 69. N 5. P. 406-411.

[7] Eskov V.M., Eskov V.V., Gavrilenko T.V., Vochmina J.V. // Moscow University Physics Bulletin. 2015. Vol. 70. N 2. P. $140-152$.

[8] Eskov V.M., Eskov V.V., Vochmina J.V., Gavrilenko T.V. // Moscow University Physics Bulletin. 2016. Vol. 71. N 2. P. 143-154.

[9] Берестин Д.К., Черников Н.А., Поскина Т.Ю., Потетюрина Е.C. // Сложность. Разум. Постнеклассика. 2016. № 1. C. 71-78. DOI: $10.12737 / 18815$.

[10] Башкатова Ю.В., Белощенко Д.В., Баженова А.Е., Мороз О.A. // Вестник новых медицинских технологий. 2016. T. 23. № 3. C. 39-46. DOI: $10.12737 / 21746$.

[11] Еськов В.В., Филатов М.А., Филатова Д.Ю., Прасолова А.A. // Сложность. Разум. Постнеклассика. 2016. № 1. C. 83-91. DOI: $10.12737 / 18817$.

[12] Еськов В.М., Зинченко Ю.П., Хадарцев А.А., Филатова O.E. // Сложность. Разум. Постнеклассика. 2016. № 2. C. 58-65. DOI: $10.12737 / 21049$.

[13] Горбань И.И. // ЖТФ. 2014. Т. 84. № 3. С. 22-30.

[14] Стёпин В.С., Еськов В.М., Буданов В.Г. // Сложность. Разум. Постнеклассика. 2016. № 3. С. 52-58. DOI: $10.12737 / 22113$.

[15] Gavrilenko T.V., Es'kov V.M., Khadartsev A.A., Khimikova O.I., Sokolova A.A. // Advances in Gerontology. 2014. Vol. 27. N 1. P. 30-36.

[16] Filatova O.E., Provorova O.V., Volokhova M.A. // Human Ecology (Russian Federation). 2014. Vol. 6. P. 16-19.

[17] Es'kov V.M., Filatova O.E., Provorova O.V., Khimikova O.I. // Human Ecology (Russian Federation). 2015. Vol. 5. P. 57-64.

[18] Garaeva G.R., Eskov V.M., Eskov V.V., Gudkov A.B., Filatova O.E., Khimikova O.I. // Human Ecology (Russian Federation). 2015. Vol. 9. P. 50-55.

[19] Karpin V.A., Filatova O.E., Soltys T.V., Sokolova A.A., Bashkatova Yu.V., Gudkov A.B. // Human Ecology (Russian Federation). 2013. Vol. 7. P. 3-9.

[20] Eskov V.M., Eskov V.V., Braginskii M.Ya., Pashnin A.S. // Measurement Techniques. 2011. Vol. 54. N 7. P. 832-837.

[21] Es'kov V.M. // Measurement Techniques. 1994. Vol. 37. N 3. P. 359-364.

[22] Es'kov V.M., Filatova O.E., Ivashenko V.P. // Measurement Techniques. 1994. Vol. 37. N 8. P. 967-971.

[23] Es'kov V.M., Filatova O.E. // Neurophysiology. 1995. Vol. 25. N 6. P. $348-353$.

[24] Eskov V.M. // Modelling, Measurement and Control C. 1995. Vol. 48. N 1-2. P. 47-63. 
[25] Es'kov V.M., Papshev V.A., Es'kov V.V., Zharkov D.A. // Measurement Techniques. 2003. Vol. 46. N 1. P. 93-99.

[26] Bernstein N.A. The co-ordination and regulation of movements. Oxford: Pergamon Press, 1967.

[27] Хадарцев А.А., Филатова О.Е., Зинченко Ю.П. // Сложность. Разум. Постнеклассика. 2016. № 3. C. 6-15. DOI: $10.12737 / 22107$.

[28] Eskov V.M. // Emergence: Complexity and Organization. 2014. Vol. 16. N 2. P. 107-115.

[29] Eskov V.M., Eskov V.V., Gavrilenko T.V., Vochmina Yu.V. // Biofizika. 2017. Vol. 62. N 1. P. 168-176.

[30] Зинченко Ю.П., Филатова О.Е., Еськов В.В., Стрельцова T.B. // Вестник новых медицинских технологий. 2016. T. 23. № 3. C. 31-39. DOI: 10.12737/21745.

[31] Филатов М.А., Филатова Д.Ю., Поскина Т.Ю., Веракca A.Н. // Сложность. Разум. Постнеклассика. 2016. № 1. C. 17-24. DOI: 10.12737/18811. 